\title{
Another Look at Provable Security
}

\author{
Alfred Menezes \\ Department of Combinatorics \& Optimization \\ University of Waterloo \\ ajmeneze@uwaterloo.ca
}

\begin{abstract}
Many cryptographers believe that the only way to have confidence in the security of a cryptographic protocol is to have a mathematically rigorous proof that the protocol meets its stated goals under certain assumptions. However, it is often difficult to assess what such proofs really mean in practice especially if the proof is non-tight, the underlying assumptions are contrived, or the security definition is in the single-user setting. We will present some examples that illustrate this difficulty and highlight the important role that old-fashioned cryptanalysis and sound engineering practices continue to play in establishing and maintaining confidence in the security of a cryptographic protocol.
\end{abstract}

This talk is based on joint work with Neal Koblitz 23. and with Sanjit Chatterjee and Palash Sarkar [1].

\section{References}

1. Chatterjee, S., Menezes, A., Sarkar, P.: Another Look at Tightness. In: Vaudenay, S. (ed.) SAC 2011. LNCS, vol. 7118, pp. 293-319. Springer, Heidelberg (2011)

2. Koblitz, N., Menezes, A.: Another look at provable security, http://anotherlook.ca

3. Koblitz, N., Menezes, A.: Another look at security definitions, Cryptology ePrint Archive: Report 2011/343 